\title{
Generic Fabrication Technique of Graphene Based RF Sensor towards Biological Application
}

\author{
Idris Musa, John Hedley
}

\begin{abstract}
Herein presented, we demonstrate that a sensitive sensing/detection element was obtained from the laser treatment of a non-conducting flexible material exploiting laser machine, which can then potentially deploy as sensing element of a biosensor for possible usage in to sense and obtain the presence and quantity of the interested sample. The goal is to study and advance innovative means of fabricating a low-cost graphene sensor, employed as a Radio Frequency (RF) filter for disposable biomedical purposes. A material like Graphene can be fashioned by laser irradiation (Laser scribe) of Kapton tape implemented as a filter. The manufacture of the filter geometry was accomplished by means of a laser machine irradiating a Kapton tape on a chosen substrate (for this work a Flame Retardant 4 (FR-4)), by the application of the previous gotten parameters for the production. Various laser power values were employed for their manufacture and their corresponding conductivity was observed to range from $171 \times 10^{-6} \mathrm{~S} / \mathrm{mm}$ to $279 \times 10^{-6} \mathrm{~S} / \mathrm{mm}$. The Raman spectrum results of the produced material has a $D$ band peak at $1349.76 \mathrm{~cm}^{-1}$, a G band of $1587.73 \mathrm{~cm}^{-1}$ and a $2 D$ band peak of $2693.34 \mathrm{~cm}^{-1}$. The ANSYS high-frequency structure simulator (HFSS) (for the Analysis of the System) simulation results signifies good outcomes, and opportunities to improve the material property are also being studied. Tests were also conducted by the utilization of a Vector Network Analyzer (VNA) to validating their feasibility of being deployed as the detection element of a biosensor, thus lending them the possibility to find implementation in disposable biological sensing.
\end{abstract}

Keywords: Laserscribe; Graphene; Biosensor; Radio Frequency filter; Kapton tape; Raman.

\section{INTRODUCTION}

In medical diagnostics it is essential to have an accurate, fast, and low-cost devices, which can be regularly employed. In this framework, biosensors are chosen to deliver a feasible answer to the difficulties presented by the modern healthcare sector. This is because they possess considerable benefits such as: specificity, minute size, cheapness, and quicker result than the laboratory test. These cheap, real, portable, and robust diagnostic devices have achieved substantial attention since there is a significant necessity for bio-devices which can speedily and accurately examine biological targets.

Manuscript received on June 15, 2021.

Revised Manuscript received on June 27, 2021.

Manuscript published on July 30, 2021.

* Correspondence Author

Idris Musa*, PhD Student, Graphene Based RF Biosensor Design and Implementation, school of Engineering, Newcastle University, England. Email: i.musa@newcastle.ac.uk

Dr John Hedley, Senior Lecturer, School of Engineering, Newcastle University, England. Email: john.hedley@newcastle.ac.uk

(C) The Authors. Published by Blue Eyes Intelligence Engineering and Sciences Publication (BEIESP). This is an open access article under the CC BY-NC-ND license (http://creativecommons.org/licenses/by-nc-nd/4.0/)
Recently, various ranges of chemicals, biochemical and biological targets have been sensed exploiting sensors derived from carbon materials.

The rate of electron transfer and the results from these devices are greatly impacted by how the carbon material itself is assembled. This is largely because of the electronic state's density, as well as the edge-plane site available on the surface of the carbon material [1,2].

There has been past research on Laser-scribe Graphene (LSG) like in [1], where in their work Graphene Oxide (GO) film was employed in the fabrication of LSG electrode, by means of light scribe Digital Versatile Disc (DVD) label writing expertise, this permits for direct laser writing of the DVD on the GO. In their work Tian et al. manufactured an elastic strain sensor utilizing a light scribed DVD burner device to reduce GO film, they accomplished a flexible and bendable graphene strain sensor possessing a low Gauge Factor (GF) when used for high a deformation application, and also a high gauge factor for a low strain sensing [3].

The scarcity of Graphene-growing and shaping methods has been a grave problem affecting its usage in numerous focused applications [3]. Graphene, can have 3-dimensional (3D) structures and sponginess, by way of synthesis and engineering strategy, making them find a wide variety of applications like: composite filter, one-use electronics, energy storing devices and elastic electronics [4]-[5]. Despite these advantages, the present synthesis scheme of porous graphene needs high temperature processing of multiple steps chemical synthesizing approaches, thus restricting their extensive profitable usage [4]. Consequently, direct synthesizing and scalability of graphene materials is still a significant technological aim in accomplishing the commercialized applications for graphene material particularly in biochemical and biological sphere [4]. Pulse ultraviolet laser treatment has been used to fashioned glassy carbon from insulating Polyimide (PI) [4, 6], and microsupercapacitor was also fashioned from commercial polymer film utilizing $\mathrm{CO}_{2}$ infrared laser [4].

Nevertheless, the method of laser irradiation for the manufacture of an RF low pass filter as a microstrip transmission line has up till now not been shown. In this research, we present a generic technique for the manufacture of a microstrip lowpass filter made of a graphene-like material that is derived by the laser reduction method, from the laser irradiation a non-conducting film which has shown promise in the biological sensing/detection application of LSG. In their work Maher et al. utilized a normal light scribe DVD optical drive to perform direct laser reduction of graphite Oxide film into Graphene [7].
Blue Eyes Intelligence Engineering and Sciences Publication (C) Copyright: All rights reserved.

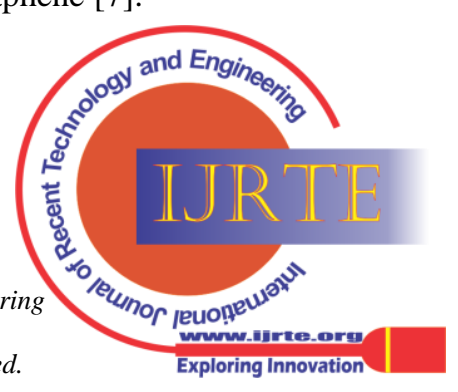


Good results for this material were indicated by their mechanical strength, electrical conductivity and specific surface area, which was then directly utilized as the electrodes of an electrochemical capacitor, where high energy density values in different electrolytes was illustrated by the devices produced from these electrodes, while preserving good electrochemical characteristics under mechanical stress that is high, making them a good candidate for high power elastic electronics [7]. All the aforementioned research has indicated the potential of an LSG material in their usage for biological and electrochemical applications, therefore, the need to design and manufacture a generic, cheaper and easily fabricated method of deriving an appropriate detection/sensing platform from LSG material. For this work, since the material for realizing a sensing/detection element is defined, the next stage of the work is to discuss the shape of the sensing element.

Research have shown that there is an increasing requirement for microwave systems to meet the evolving problems facing telecommunication with respect to cost, performance, and size [8]. This research seeks to define the design of a microstrip lowpass RF filter for biological application. The microstrip is an electrical transmission line that can be manufactured utilizing a Printed Circuit Board (PCB) technology deployed to transmit microwave signal [8]. This microstrip contains a conductor detached form a ground plane by a dielectric substrate material and it is much inexpensive than the waveguide technology [8]. The microstrip for this work will be designed as a lowpass filter, since filters are critical parts in a microwave communication systems and RF circuitries, they are employed to combine or separate the chosen frequencies in the band, to fulfil the essential requirement [9].

The microstrip line is a good method for designing filter due to its advantages of cheapness, light weight, compacted size, planar structure and how it can easily combine with other component on a single board [10].

In addition microwave filters can be separated in to 2 different categories; the first being the lumped element, comprising capacitors and inductors and the second being the distributed element, which employs transmission lines lengths and widths to produce their inductance or capacitance value $[10,11]$, leading to an Inductor-Capacitor (LC) ladder type stepped impedance Low Pass (LP) filter, if a LPF was the design proposed [12]. This work is structured as follows: Section II is concern with the microstrip analytical design, while section III indicates the experimental approaches. the results were illustrated in section IV, and section $\mathrm{V}$ deals with the discussion and conclusion.

\section{DESIGN PROCEDURES}

\section{A. The Design of a Microstrip Transmission Line}

Numerous diverse equations have been established for use in the determination of the characteristic impedance of microstrip design. Possibly the most expedient are the highlighted ones below that are stated to be precise to about $1 \%$ [12]. A simple but precise equation for the characteristic impedance of the microstrip is presented below with Fig 1 representing the microstrip structure [13]:

$$
Z 0=\frac{60}{\sqrt{\varepsilon e f f}}\left\{\log \left(8 \frac{h}{w}+\frac{w}{4 h}\right)\right\}
$$

where the effective dielectric constant is given by (2) as:

$$
\varepsilon e f f=\frac{\varepsilon r+1}{2}+\frac{\varepsilon r-1}{2}\left\{\left(1+12 \frac{h}{2 w}\right)^{-1 / 2}+0.04\left(1-\frac{w}{h}\right)^{2}\right\}
$$

while (3) and (4) are for when $w / h \geq 1$

$$
Z 0=\frac{120 \pi}{\sqrt{\varepsilon e f f}\left\{\frac{w}{h}+1.393+0.667 \log \left(\frac{w}{h}+1.444\right)\right\}}
$$

where:

$$
\varepsilon e f f=\frac{\varepsilon r+1}{2}+\frac{\varepsilon r-1}{2}\left\{\left(1+12 \frac{h}{2 w}\right)^{-1 / 2}\right\}
$$

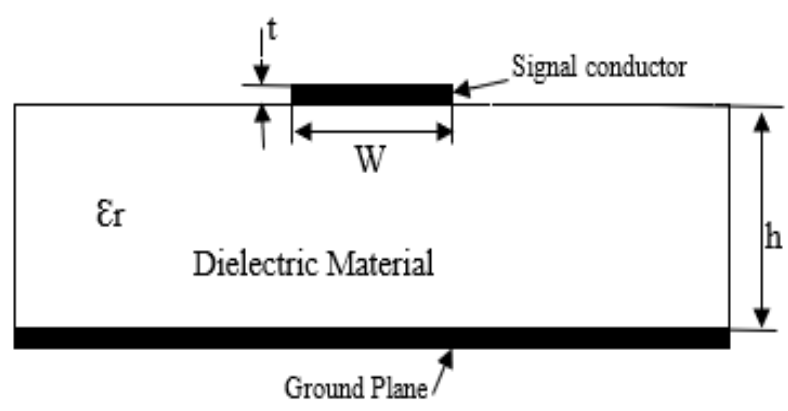

For this research, the microstrip design was based on the second sets of circumstances where $w / h \geq 1$, therefore equations (3) and (4) was utilized.

\section{B. A Single Strip Case Microstrip Transmission Line Design}

From equations (3) and (4), the proposed microstrip line width was then determined, since for a wide microstrip where width $=\mathrm{W}$, virtually all the electric field lines will be directed between the metal planes, like the situation of a parallel plate capacitor. Conversely, for narrow width the electric field lines will be about evenly shared between the board dielectric and air [12].

To acquire the effective dielectric constant of a microstrip transmission line, equation (4) should have been used, nevertheless, the equation have 2 unknown parameters, consequently, the formula that presents an estimated value of the characteristics impedance was then employed, which is presented in equation (5) $[11,14]$.

From which:

$$
Z 0=\frac{87}{\sqrt{\varepsilon r}+1.41}\left\{\log \left(\frac{7.48 h}{w-1.25 T}\right)\right\}
$$

$$
w=\frac{7.48 h}{e^{\left(z 0 \frac{\sqrt{\varepsilon r}+1.41}{87}\right)}}+(1.25 t)
$$

For this research work, an FR-4 dielectric material was utilized as the substrate, with a dielectric constant of 4.4 [11, 15]. Results from the pre-test samples shows that the thickness $\mathrm{T}$ was found to be $4.8532 \mathrm{mil}$ in dimension. The FR-4 board of dielectric material used for this work has a thickness of $10 \mathrm{mil}$. While the material characteristic impedance of choice is $50 \Omega$. The following was deduced from the information above: $\mathrm{T}=4.8532 \mathrm{mil}, \mathrm{H}=10 \mathrm{mil}$ $\mathrm{Z} 0=50 \Omega \varepsilon r=4.4$.

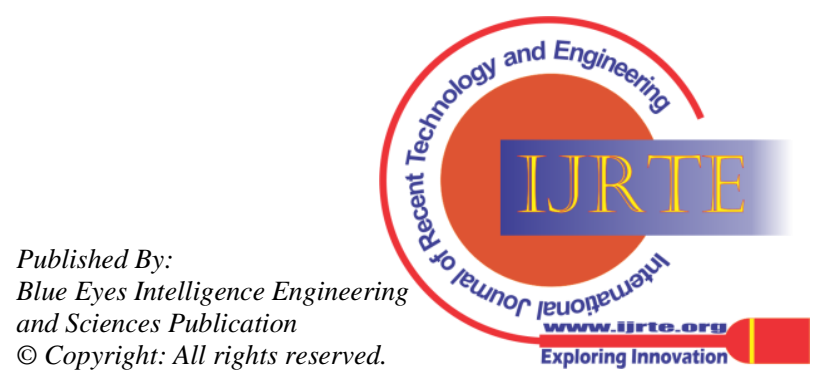


The width of the material was be calculated as shown below by inserting all these parameters in equation (6) to be $\mathrm{w}=0.635 \mathrm{~mm}$. This calculated value is closer in value to the measured value when a Mitutoyo stylus surface roughness measuring tester (Surftest SJ-410) was utilized, where the observed thickness was measured to be $0.4835 \mathrm{~mm}$.

\section{A Multiple Strip Case Microstrip Transmission Line Design}

This multiple strip material is made up of one strip comprising several single strips fabricated near each other, consequently, the estimation of its width is simply the summation of the width of the distinct single strip and their resultant sum becomes the width of this strip. However, the observed out produced a different result because during this material production it was detected that the laser machine normally produce overlapping strips on the Kapton tape which suggest that the width of the produced material would not be brought about as expected and explained above. Consequently, this material width was not calculated but rather measured using the Surftest SJ-410, the width obtained from this measurement was recorded as $1.75875 \mathrm{~mm}$ which corresponds to70 mil. To acquire the precise results for each of the slope of the measured results of the resistance of the fabricated material along its length, an analytical technique for the determination of the slope of the straight line was employed. This was achieved by employing the method of least squares.

\section{EXPERIMENTAL TECHNIQUE}

The analytical strategy of this microstrip complete in the preceding segment that has the evaluation (Analytical preparation) of the breadth plus predictable depth was completed. The execution of this strategy was accomplished exploiting the laser scribe method for producing GO. This method of GO manufacture is a cheap technique of manufacturing Graphene. This method is a new means of Graphene Oxide manufacture and by extension a material like graphene Oxide. To accomplish this numerous experimentations were thus implemented, for this research the initial materials were Kapton tape (on which the material of choice would be derived from), silver paint (to help ensure electrical connections between the sensor device and test equipment), plywood (for the substrate of pre-test samples), digital multimeter (to measure the conductivity of the material) and laser machine (Laserscript HPC laser, for the irradiation of the Kapton tape to form the rGO) this laser machine has a laser spot of $0.2 \mathrm{~mm}$. For comparison and to get enough information about the material two material categories were implemented, where 8 samples of the fabricated materials possessing distinct parameters were then fashioned. These categories are Multiple-Strips and Single Strip. The speed of laser engraving and the strips width were the two parameters that were varied.

The Multiple-Strips (MS) material has four set, wherein respective sample has five fabricated materials derived from the variation of the percentage laser power settings from $26 \%$ to $34 \%$ in steps of $2 \%$. This technique of fabrication was also used to implement the single strip (SS), this SS has the similar production methods having same parameters as the MS material, however distinct with the quantity of strip on it. For the MS, the two parameters of the laser "engraving speed" and the strip "width" were selected and established. The parameters change from one device sample to the other leading to the production of all the 4 sample sets for each group of the sample. The same methods used for producing the MS was also used in implementing the SS samples. For the MS and the SS, the main parameters are presented in Table I. These sets of settings deployed for both strip lines, resulted in samples equaling 8 in total. Accomplishing the samples design several procedures were followed:

1.Initially, haven done the analytical design, suitable amount of the Kapton tape material was transferred by taping unto the top of FR-4 substrate.

2.The next stage was doing the setting of the category of interest was done on the computer system.

3. The next step was doing the setting for the fabrication of the material of interest on the system, which was then copied to an external flash drive and then transferred to the laser machine awaiting the next step.

4. The next step was the introduction of the sample obtained from the first step above into the laser machine working area for the commencement of the laser engraving procedure.

5. To produce the entire 40 samples the manufacture procedure from step 1 to step 4 above was repeated for all the 2 classes of material.

With the aid of a digital multimeter the needed resistance values from the fabricated sample devices were obtained. The motive behind determining the resistance value is to aid the ascertaining the resistivity of the resultant material, so as to check their feasibility of being deployed as a material that possess the necessary property leading to their usage as the sensing/detection element of a biosensor.

TABLE-I. Settings to Produce the Microstrip

\begin{tabular}{|l|l|l|l|l|}
\hline parameter & Sample 1 & Sample 2 & Sample 3 & Sample 4 \\
\hline Speed $(\mathrm{mm} / \mathrm{s})$ & 300 & 300 & 400 & 400 \\
\hline Width $(\mu \mathrm{m})$ & 300 & 350 & 300 & 350 \\
\hline \multirow{2}{*}{ \% power } & $\begin{array}{l}26, \quad 28, \\
30, \quad 32,\end{array}$ & $\begin{array}{l}26,28,30, \\
34,\end{array}$ & $\begin{array}{l}\text { 26, 28, 30, } \\
32,34 .\end{array}$ & $\begin{array}{l}\text { 26, 28, 30, 34. } \\
\text { 34. }\end{array}$ \\
\hline
\end{tabular}

of the projected biosensor device. Test were carried out on the 2 classes of materials (MS and SS), this is to explore which of the sample from any of the 2 sets of samples (20 different materials for the multiple strip and 20 the single strip) is workable platform and results were taken. The tests results were recorded.

Emanating from these results were several plots being done with results processing, with further operations it then resulted in determining the material associated parameters and that is optimal and appropriate for the material of interest design. The area of each material is required to be determined, this is so that the apt resistivity of the materials produced, can be arrived at, and to accomplish this task, the individual material thickness and the width were measured employing the Mitutoyo stylus surface roughness measuring tester (Surftest SJ-410), armed with this device the device measurement was done to the nearest micrometer. This method was then utilized to produce a $5^{\text {th }}$ order LPF microstrip LSG on a PCB substrate for deployment in the proposed application.

Blue Eyes Intelligence Engineering and Sciences Publication

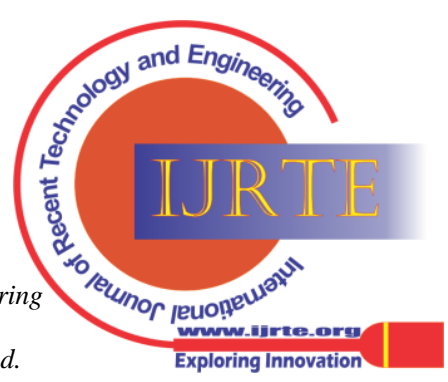


Below is Fig 2 which gives the fabrication procedure for the phase-by-phase process of implementing the LSG microstrip LPF, whereas Fig 3 illustrates the production machine with the filter during fabrication process.

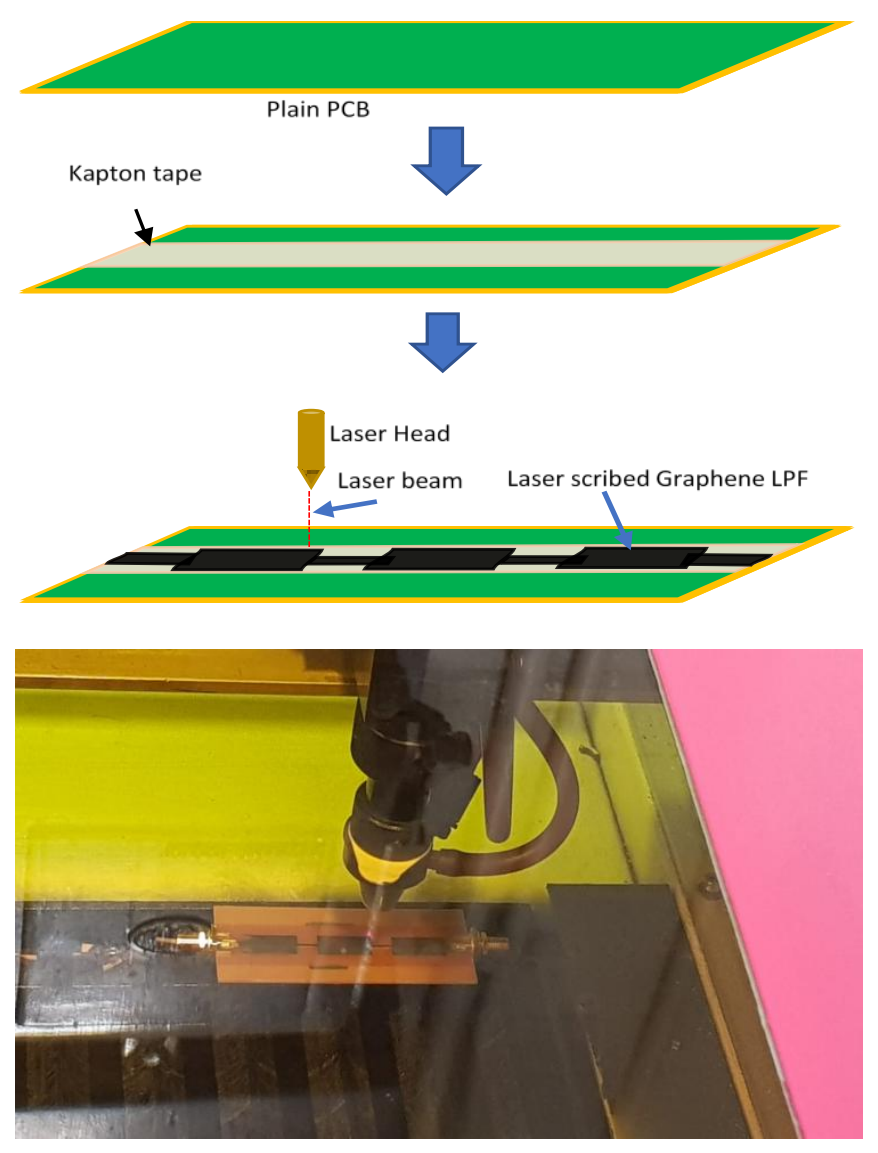

\section{THE SAMPLE RESULTS}

The test results undertaken for the distinct parameters of the various materials developed, were noted during their measurement. Scrutinizing the obtained results, it was detected that, certain measured values of resistance do not proportionally rise linearly with a corresponding increase in length, thus the behavior are considered be irregular. Consequently, this point to the material amongst the sets of samples that could possess the desirable characteristic that makes a viable sample for the proposed application. Further processing of these results led to the utilization of plots to obtain the slope, which is then the resistance per unit length of the fabricated material. Fig 4 illustrates a typical slope for these measured results. Following the same procedure, the results of the single strip line was also obtained while employing the same equipment as those utilized for the multiple strips above, the results were gotten and recorded as shown below. These results enable plots to be made leading to the determination of the slope of the result which is also called the resistance per unit length of the manufactured material. A typical slope results are presented in Fig 5 below.
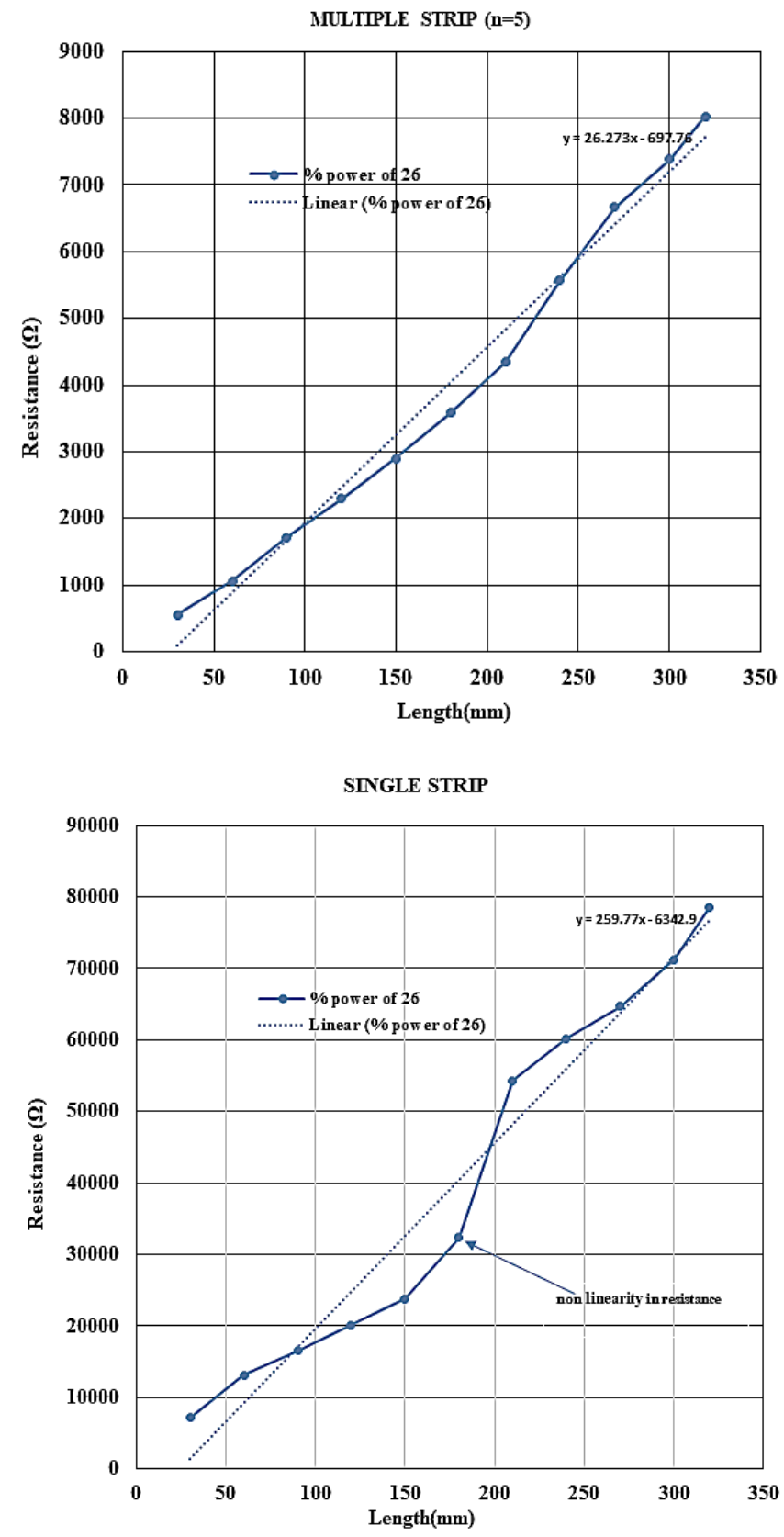

From the presented results, a plot of the resistance per unit length was done against the percentage power in both material categories. These results are presented in Fig 6a and 6b. Evidence from these plots illustrate that the SS materials is obviously characterized with more associated discontinuity in terms of material conductivity. Thus, the specific materials and the corresponding percentage powers where these discontinuities happened has become evident from these plots. As can be observe from these plots certain points were specifically enlarged, the essence of which is to accentuate the materials with detected anomalies as well as the exact percentage powers which they were manufactured. Also important about these plots is that they have made it easier in identifying the material with the desirable characteristics from either class of materials for ease of material selection.

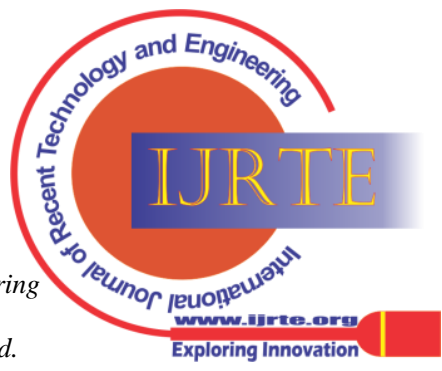


As is evidence from the results presented in Fig 6, in the plot of fig $6 \mathrm{a}$ and $6 \mathrm{~b}$, it showed that the value of the resistance per unit length for the SS material (which is on the vertical axis of this plots) is about 10 times the value of the resistance per unit length for the MS material. Furthermore, the number of discontinuous/defected materials as illustrated by highlighted black dots are predominant found in the single strip than the multiple strip materials. On the whole these results have shown that multiple strip material has revealed interesting characteristics that make their design approach viable for it to be employed for the design of the proposed microstrip lowpass filter, that would then become the sensing/detection element of the proposed biological sensor whose effectiveness is reliant on the material resistivity. In an effort to arrive at the most effective fabrication approach that is suitable for producing this laser irradiated graphene Oxide material, additional results processing was carried out, consequently resulting in plots of Fig 6c and 6d. The results demonstrate typical plot for either category, out of which valuable outcomes about each material and the fabrication technique of producing each material can be derived. Simulation using ANSYS HFSS software was carried out by applying the conductivity value obtained from the test results conductance, and these simulation results showed that the manufactured filter's response was comparable to that of the theoretical lowpass filter presented in Fig 8.

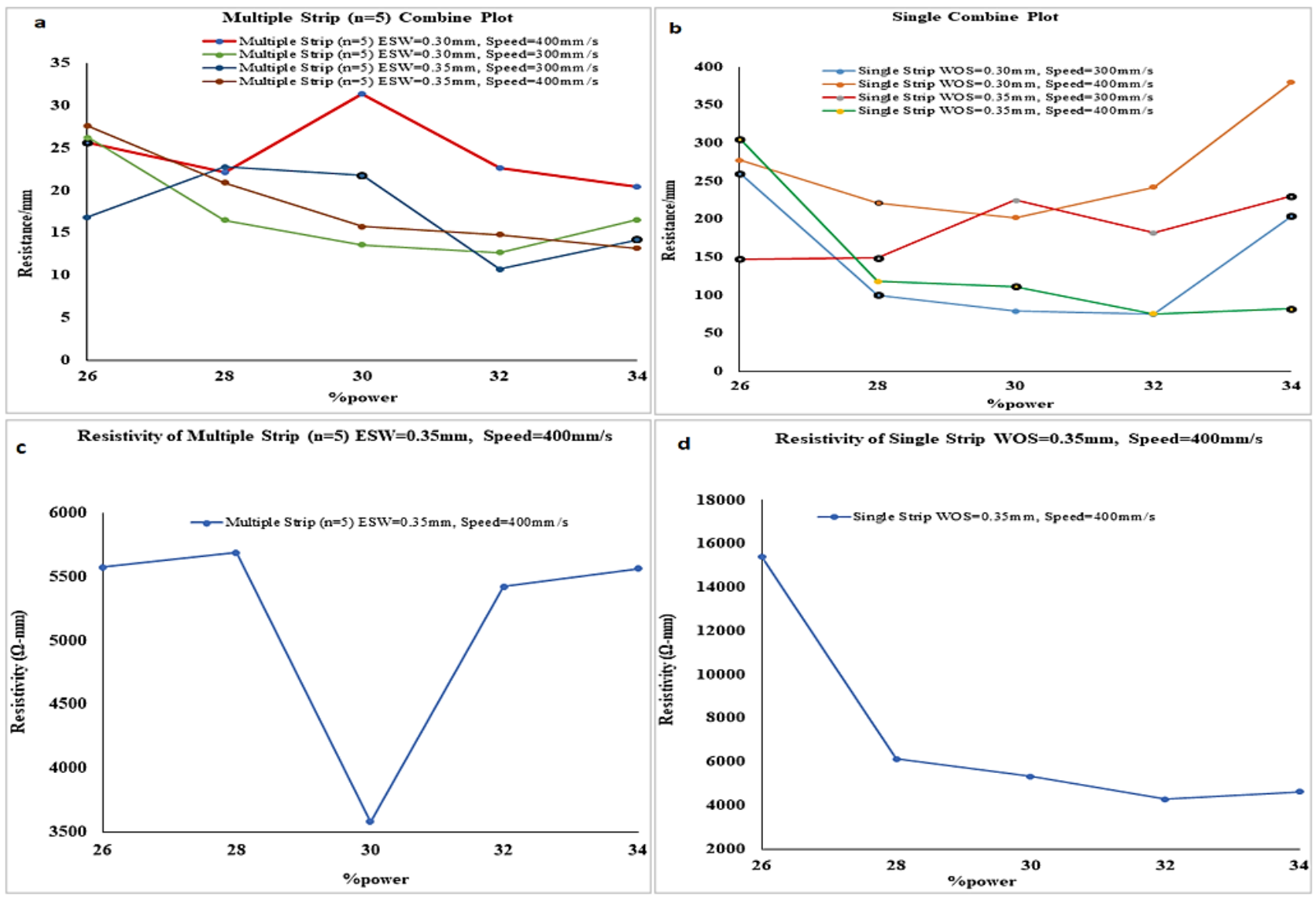

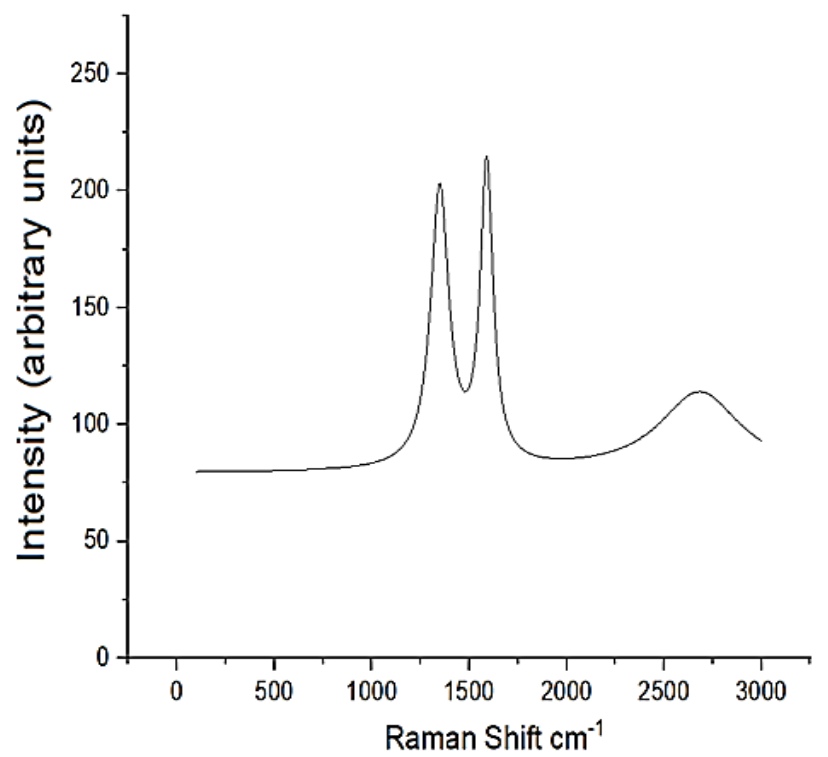

Retrieval Number: 100.1/ijrte.B61200710221

DOI: 10.35940/ijrte.B6120.0710221

Journal Website: www.ijrte.org
A Raman spectrum of a solid contains evidence about its vibrational and electronic properties [16]. Raman spectroscopy is the tool of choice in the physicochemical investigation of carbon nanomaterials. It is among the primary techniques for the characterization of graphene materials, as it provides insights into the quality of measured graphene including their structure and conductivity as well as the presence of dopants. The Raman intensity and Raman band shape vary as functions of the number of graphene layers, and the Raman 2D band is especially sensitive to the number of graphene layers [17].

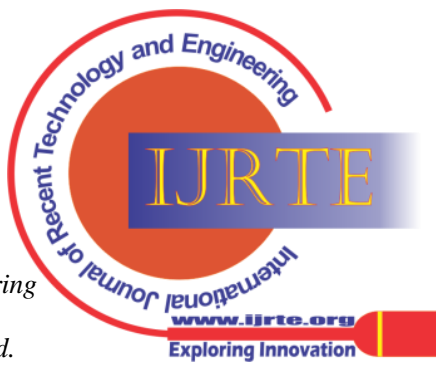


From the above Raman result which represents the Raman spectrum of the material whose parameters is presented in fig. 7 above, the rGO derived from the laser irradiated rGO (by laser reduction of Kapton Tape) was observed to have a D band peak at $1349.76 \mathrm{~cm}^{-1}$, a G band of $1587.73 \mathrm{~cm}^{-1}$ and a 2D band of $2693.34 \mathrm{~cm}^{-1}$. Comparing these results with the result of the Rama spectrum of the graphene obtained with the same laser excitation of 532nm used for the material produced in this work, it was noted that in the work of Bing Ma et al in [18], and Isaac Childres et al in [19] for graphene the value of the $D$ band was reported to be $1350 \mathrm{~cm}^{-1}$, the $G$ band is $1580 \mathrm{~cm}^{-1}$ and 2D band to be $2690 \mathrm{~cm}^{-1}$. From these results it can be observed that the Raman spectrum of both graphene and the rGO here produced tend to be show closely related value, thereby indicating that the rGO material also have low number of graphene layers, thus making the rGO material a potential candidate for the implementation of devices for various electrical and electronics application, consequently making them open for biomedical and biochemical applications.
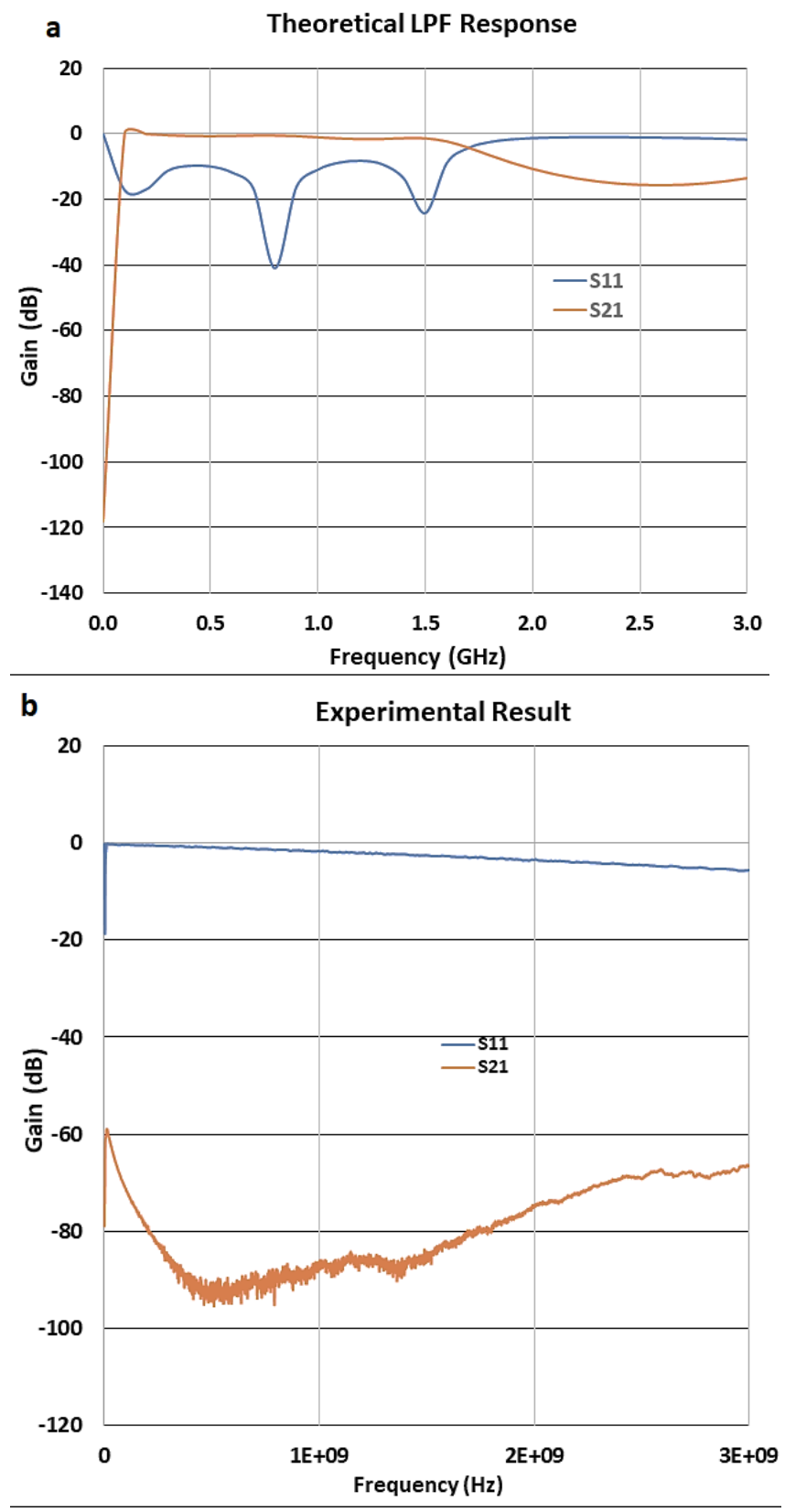

Haven obtained the Raman spectrum the produced material was then used to implement a $5^{\text {th }}$ order lowpass filter to verify its viability for biological applications. Haven produced the $5^{\text {th }}$ order LPF using the MS line technique. VNA was used to measure its S-parameters, to determine suitability for the intended application. From the observed results shown in Fig. 8, there is a difference between the expected and the experimental/simulation response of a microstrip LPF, thereby indicating that more fine-tuning of the fabrication parameters is necessary. However, the Raman response indicate few numbers of layers, thus, making them a potential candidate for the intended RF biomedical application.

\section{CONCLUSION}

The aim of this work was to develop new ways of producing a graphene base RF sensor, starting with cheap rapid prototyping of implementing an inexpensive and flexible electronic material that is easily reproduceable, with high conductivity, and can be deployed for use as a sensor for biological application. The Raman spectrum results of the produced material has a D band peak at $1349.76 \mathrm{~cm}^{-1}$, a G band of $1587.73 \mathrm{~cm}^{-1}$ and a 2D band of $2693.34 \mathrm{~cm}^{-1}$. While the conductivity results indicated that the multiple strip line material has a promising conductivity value ranging from $171 \times 10^{-6} \mathrm{~S} / \mathrm{mm}$ to $279 \times 10^{-6} \mathrm{~S} / \mathrm{mm}$, signaling its viability for the proposed application. From these results, it can be concluded that this LIG can also find application in various sensing platforms where light weight and flexible conductor is desired. The results of the microstrip LPF implementation indicated the need to fine tune fabrication parameters so as to improve the filter response. The future work includes fine tuning the fabrication parameters and the use as a biological sensor. Then, testing the device with a mouse IgG needs to be done, to show its applicability as a biological sensor before moving on to more sensitive analytes like Prostate-specific antigen (PSA). To achieve that, in this work, both a Chebyshev type $5^{\text {th }}$ and $7^{\text {th }}$ order low pass and bandpass filters are to be implemented as the sensing surface using the insertion loss method.

\section{ACKNOWLEDGMENT}

This work was sponsored by Petroleum Technology Development Fund (PTDF). We also thank Frank Atkinson for his support.

\section{REFERENCES}

1. K. Griffiths, J.H. C. Dale, and R.B.K. M. D. Kowal, N. Keegan, , Laser-scribed graphene presents an opportunity to print a new generation of disposable electrochemical sensors. Nanoscale, 2014. 6(22): p. 13613-22.

2. R. L. McCreery, a.M., M. T., Comment on electrochemical kinetics at ordered graphite electrodes. Anal Chem, 2012. 84(5): p. 2602-5.

3. H. Tian, et al., Scalable fabrication of high-performance and flexible graphene strain sensors. Nanoscale, 2014. 6(2): p. 699-705.

4. J. Lin, et al., Laser-induced porous graphene films from commercial polymers. Nat Commun, 2014. 5: p. 5714.

5. Z. Chen, et al., Three-dimensional flexible and conductive interconnected graphene networks grown by chemical vapour deposition. Nat Mater, 2011. 10(6): p. 424-8. 
6. M. Schumann, R. Sauerbrey, and M.C. Smayling, Permanent increase of the electrical conductivity of polymers induced by ultraviolet laser radiation. Applied Physics Letters, 1991. 58(4): p. 428-430.

7. El-Kady, M., et al., Laser Scribing of High-Performance and Flexible Graphene-Based Electrochemical Capacitors. Science (New York, N.Y.), 2012. 335: p. 1326-30.

8. K. Rajasekaran, J. Jayalakshmi, and J. Thangaiyan, Design and Analysis of Stepped Impedance Microstrip Low Pass Filter Using ADS Simulation Tool for Wireless Applications. 2013. 3: p. 1-5.

9. Nur Baya Mohd Hashim, M.S.R., Siti Zuraidah Ibrahim and Fazlina Farid, Compact Ultra Wideband Stepped Impedance Low Pass Filter Utilizing Open Circuit Stub Resonator. ARPN Journal of Engineering and Applied Sciences, 2015. 10: p. 9120-9126.

10. Islam Mansour, H.E., and Adly S. Tag El-dein, Design of Stepped Impedance Microstrip Low Pass Filter with DGS. International Journal of Engineering Research and Development, 2014. 10(7): p. 58-67.

11. Anerao, M.V.M., Implementation of Stepped Impedance Microstrip Low Pass Filter. International Journal of Emerging Trends in Electrical and Electronics (IJETEE) 2013. 2(2): p. 89-92.

12. Garvansh, N.S.a.A.K., Design and Synthesis of Stepped Impedance Microstrip Line Low Pass Filter. International Journal of Scientific Research Engineering \& Technology, 2014: p. 48-50.

13. Rosu, I., Microstrip Stripline and CPW Design, , http://www.qsl.net/va3iul.

14. Hall, S.H., G.W. Hall, and J.A. McCall, High Speed Digital System Design A Handbook of Interconnect Theory and Design Practices. 2000.

15. Wang, C., Determining Dielectric Constant and Loss. 2000.

16. Graf, D., et al., Raman imaging of graphene. Solid State Communications, 2007. 143(1-2): p. 44-46.

17. Duhee Yoon, H.M., Hyeonsik Cheong, Jin Sik Choi, Jung Ae Choi and Bae Ho Park, Variations in the Raman Spectrum as a Function of the Number of Graphene Layers. 2009.

18. Ma, B., et al., The correlation between electrical conductivity and second-order Raman modes of laser-reduced graphene oxide. Phys Chem Chem Phys, 2019. 21(19): p. 10125-10134.

19. Isaac Childres, L.A.J., Wonjun Parkb, Helin Cao and Yong P. Chen, RAMAN SPECTROSCOPY OF GRAPHENE AND RELATED MATERIALS.

\section{AUTHORS PROFILE}

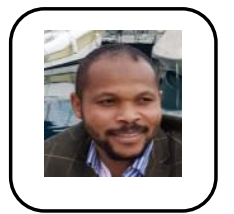

Idris Musa, is a $\mathrm{PhD}$ student on Graphene based $\mathrm{RF}$ Biosensor design and implementation with Newcastle University in the school of Engineering, with areas of expertise in Sensor, MEMS, biosensors, and 2D materials. He acquired his MSc in Automation and Control from Newcastle University in 2013 and his BEng in Electrical and Electronics Engineering in 2007 from University of Maiduguri. A lecturer with Abubakar Tafawa Balewa University.

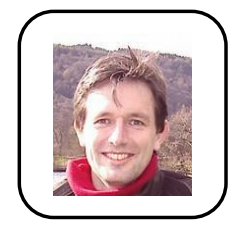

Dr John Hedley, is a Senior Lecturer for the School of Engineering at Newcastle University, with areas of expertise in MEMS, biosensors, and graphene. $\mathrm{PhD}$ Collisional and radiative processes in atomic spectra. BSc (Hons) - Astronomy and astrophysics. Previous: Director of Postgraduate Studies, School of Mechanical and Systems Engineering (2009 - 2013) MSc Microsystems Engineering Degree Programme Director (2007 - 2014), MSc Biomedical Nanotechnology Degree Programme Director (2004 2006). Current: MSc Mechatronics Degree Programme Director (2014 present).

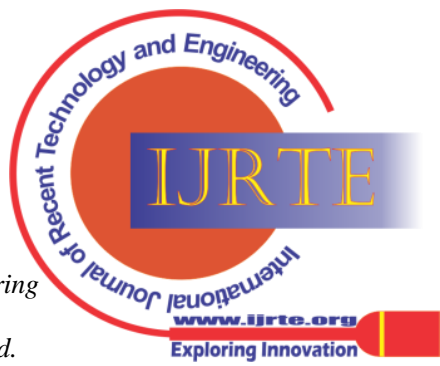

